\title{
A ROCKET-BORNE X-RAY SPECTROMETER/ MONOCHROMATOR SYSTEM FOR MAPPING THE SOLAR CORONA
}

\author{
L. W. ACTON, R. C. CATURA, J. L. CULHANE, and A. J. MEYEROTT \\ Lockheed Palo Alto Research Laboratory, Palo Alto, Calif., U.S.A.
}

A rocket payload is being prepared for the purpose of examining the spatial distribution of line emission from two important ions, O VII and NeIX, in the solar corona. The payload will contain the following integrated set of instruments.

(1) A pair of X-ray spectrometers utilizing KAP crystals of approximately $100 \mathrm{~cm}^{2}$ area.

(2) An optical aspect camera with a $1 \AA$ bandpass $\mathrm{H}-\alpha$ filter to measure the location of the field of view of the X-ray systems on the sun through out the rocket flight.

(3). A collimated proportional counter spectrometer operating in the 3 to $15 \mathrm{keV}$ range.

The two X-ray analysing crystals will view the Sun through collimators which limit their field of view to 1.7 arc min FWHM. Each crystal will be sequentially positioned so as to scan over a narrow wavelength range once each second in 64 steps. The two spectrometers will cover the wavelength intervals containing the helium-like emission lines of O vII (21.6 $\AA$ ) and NeIX (13.5 $\AA$ ) respectively. The diffracted X-rays will be detected by thin-window proportional counters and the accumulated counts will be processed and sent to telemetry by a digital data processor in synchronism with the stepwise crystal scan.

During the rocket flight the SPARCS attitude control system will cause the field of view of the X-ray systems to traverse a $40 \mathrm{arc}$ min path centered on the Sun at an angular rate of approximately 0.33 arc min per second, roll the payload approximately $90^{\circ}$ about its longitudinal axis, and then execute a second 40 arc min traverse of the Sun. The spatial traverse rate will allow about $5 \mathrm{~s}$ of observing time (corresponding to 5 wavelenth scans by the analysing crystals) per 1.7 arc min on the Sun. The resulting spatially and spectrally resolved data will be used to construct spectroheliograms in each of the 6 spectral lines covered by the spectrometers. The line intensity ratios will be used to compute coronal densities and temperatures.

This program is being carried out under the support of the National Aeronautics and Space Administration (contract NASw-1834) and the Lockheed Independent Research program. 